\title{
A technology to improve formability for aluminum alloy thin-wall corrugated sheet component hydroforming
}

\author{
Lihui Lang, Deshuai Konga , Zhiying Sun, Shangwen Ruan, and Shanshan Gu \\ School of Mechanical Engineer and Automation, Beihang University, Beijing 100191, China
}

\begin{abstract}
The explosively forming projectile (EFP) had been traditional adopted for the aluminum thin-walled corrugated sheet, whose deformation range is large but the formability is poor, and this process usually has problems of poor surface quality, long manufacturing cycle and high cost. The active hydroforming process was suggested to solve these issues during EFP. A new technology named as blank bulging by turning the upside down active hydroforming technology was proposed to overcome difficulties in non-uniform thickness distribution and cracking failure of corrugated sheet during the conventional hydroforming process. Both numerical simulations and experiments were conducted for this new technology. The result show that the deformation capacity of aluminum alloys can be improved effectively, and the more uniform distribution of wall thickness was obtained by this new method. It is conducted that the new method is universal for thin-walled, shallow drawing parts with complex section.
\end{abstract}

\section{Introduction}

Aluminum alloy corrugated sheet, which has the advantages of light weight, excellent bearing capacity and high performance in energy absorption, is widely used in aerospace structures [1]. However, due to its low formability at room temperature, aluminum alloy sheet is easily fractured in conventional stamping process. The explosively forming projectile (EFP) had been traditionally adopted for forming of the aluminum thin-walled corrugated sheets. Since the use of EFP is constrained due to the limitations of space, work conditions and a high requirement for vacuum degree, all of which are essentially required to get good surface quality parts. On the other hand, hydroforming is an advanced forming technology in which the flexible liquid medium is used to replace the traditional rigid die. It has the advantages of saving process time, tooling costs and can produce good quality of forming parts. In the light of the different functions of the liquid, sheet hydroforming technology is mainly divided into two categories: active sheet hydroforming and passive sheet hydroforming [2]. Active sheet hydroforming use hydraulic pressure as the main driving force to deform the blank sticks on the die.

With the application of lightweight materials such as aluminum alloys in hydroforming, research on the deformation of aluminum alloy has become more urgent. Lang [3] researched on hydromechanical

\footnotetext{
${ }^{\text {a }}$ Corresponding author: Deshuai Kong@deshuai_kong@126.com
}

This is an Open Access article distributed under the terms of the Creative Commons Attribution License 4.0, which permits unrestricted use, distribution, and reproduction in any medium, provided the original work is properly cited. 
Table 1. Material mechanical property parameters.

\begin{tabular}{|c|c|c|c|c|c|}
\hline Material & $\begin{array}{c}\text { Yield } \\
\text { strength } \\
\sigma_{s} \text { (Mpa) }\end{array}$ & $\begin{array}{c}\text { Ultimate } \\
\text { strength } \sigma_{b} \\
\text { (MPa) }\end{array}$ & $\begin{array}{c}\text { Hardening } \\
\text { coefficient, K } \\
\text { (MPa) }\end{array}$ & $\begin{array}{c}\text { Strain } \\
\text { hardening, } \mathbf{n}\end{array}$ & $\begin{array}{c}\text { Anisotropy } \\
\text { coefficient }\end{array}$ \\
\hline $\begin{array}{c}\text { Aluminum } \\
\text { alloy 2024-o }\end{array}$ & 65.67 & 178.67 & 323.35 & 0.2075 & 0.7183 \\
\hline
\end{tabular}

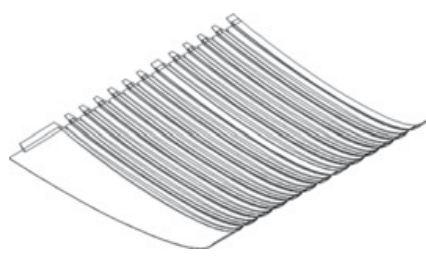

Figure 1. Corrugated sheet model.

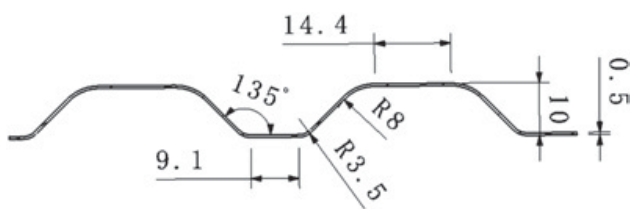

Figure 2. Sectional dimension.

deep drawing with uniform pressure onto the blank. He applied the method how to load uniform pressure. Through optimizing the process parameters, obtained material Al1050-H0 cylindrical parts whose drawing ratio is 3.11. Huseyin [4] increased the drawing ratio form 2.65 to 2.787 of aluminum ally AA5754-O by optimizing pre-bulging pressure and dimension of drawbead. Meng Bao [5] formed a box part with super wide flange using hydrodynamic deep drawing and the process window of cavity pressure was established through stress analysis of the corner and straight regions. These analytical models provided rapid and reasonably accurate results for the design of warm hydroforming process. All the above mentioned studies focus on the hydrodynamic deep drawing of aluminum alloy and innovation. However, compared with the hydrodynamic deep drawing, active hydroforming process is relatively simple and therefore, in-depth research on this process is not available. In this paper, the stress state of aluminum alloy thin-walled corrugated sheet in active hydroforming process has been analyzed, and the blank bulging by turning the upside down hydroforming method was presented to overcome the cracking failure.

\section{Forming process analysis}

\subsection{Part character and used material}

In this study, a typical corrugated sheet component whose shape and basic dimensions are shown in Fig. 1 and Fig. 2 was investigated. The AA2024-O aluminum sheet with an initial thickness of $0.5 \mathrm{~mm}$ was used, and its mechanical properties are listed in Table 1.

\subsection{Analysis of deformation process}

Considering the geometrical features of the component, active hydroforming was adopted to form it. Under the action of internal pressure, plastic deformation occurred according to the shape of the punch and the final part was formed. The deformation process of blank can be divided into 3 stages as shown in Fig. 3: the blank bending along the underside round corner of the punch $\{(\mathrm{a})$ to (b) $\}$; free bulging $\{(b)$ to (c) $\}$ and filling the upside round corner of the punch $\{(d)$ to (e) $\}$. Strain in the width direction was ignored and a plain strain state of the blank was assumed during the analysis.

Due to the shape characteristics of the corrugation unit, it was divided into 5 zones to facilitate the analysis process as shown in Fig. 4. 


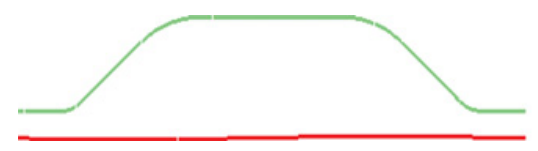

(a)

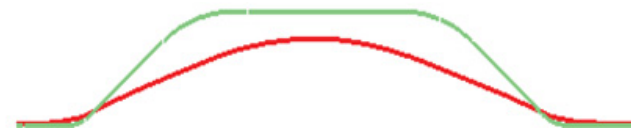

(b)

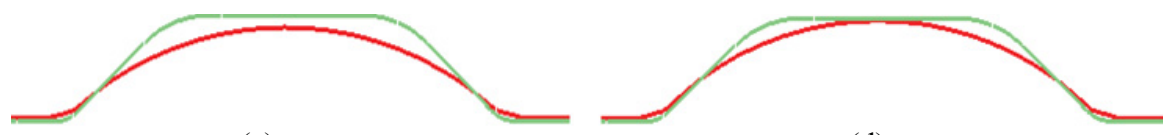

(c)

(d)

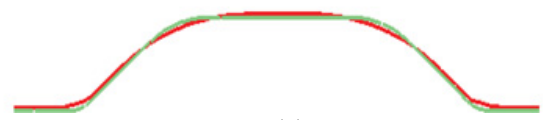

(e)

Figure 3. Deformation process of a corrugation unit.

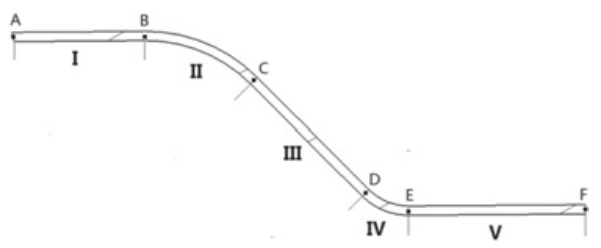

Figure 4. Typical corrugated unit.

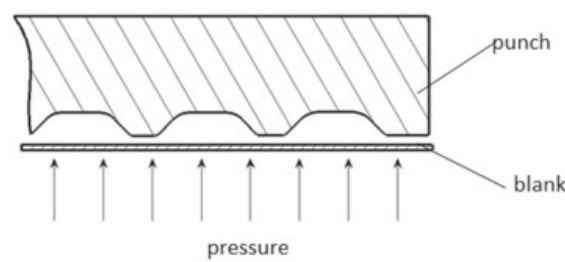

(a)

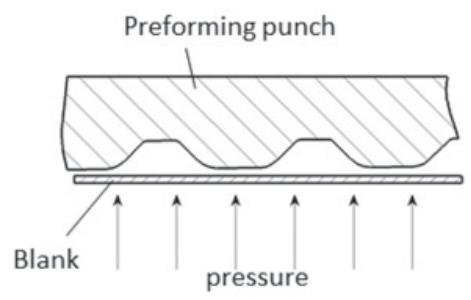

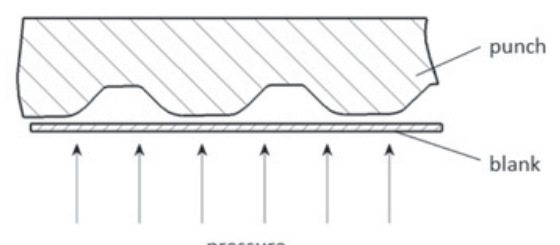

(b)

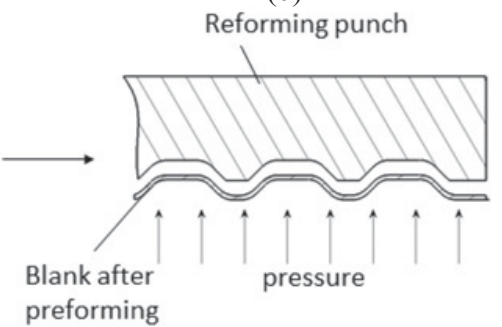

(c)

Figure 5. Schematic of forming schemes.

\subsection{Design of forming scheme}

Three active hydroforming schemes are illustrated in Fig. 5 to demonstrate: a) under the action of pressure and punch the rounded corner R3.5 is bended first, then it fills the rounded corner R8; b) under the action of pressure and punch the rounded corner R8 is bended first, then it fills the rounded corner 


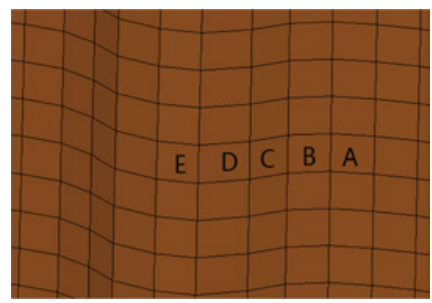

Figure 6. The finite element meshes of each region on corrugated unit.

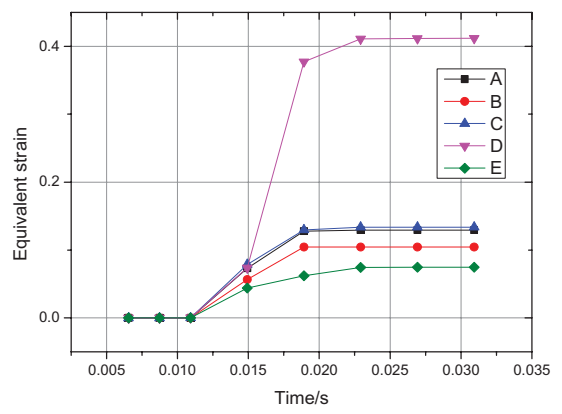

Figure 7. Effective strain variation curves of each region in forming process.

$\mathrm{R} 3.5$; c) bending the rounded corner R8 using the preforming tool with lower pressure, then turning the blank upside down and forming the rounded corner R3.5 by the re-forming tool.

The internal pressure needed in the process of rounded corner filling of hydroforming is:

$$
p_{c}=\frac{t}{r_{c}} \sigma_{s}^{\prime} \text {. }
$$

Where $r_{c}$ is radius of the minimum transition round corner; $\mathrm{t}$ is the average thickness of transition round corner; $\sigma_{s}^{\prime}$ is the flow stress in hydroforming, $\sigma_{s}^{\prime}=\frac{\sigma_{s}+\sigma_{b}}{2}$ usually.

According to formula (1), we can estimate the minimum pressure required during the hydroforming process. During present study, for case (a) it is $7.5 \mathrm{MPa}$ and for case (b) it is $17.3 \mathrm{MPa}$. While considering the area of the blank, the tonnage needed in case (b) exceeds the available capacity of the equipment. However, in case (a), during bending the round corner R3.5, the blank has a tendency to rupture because the radius is too small. Therefore, in order to overcome the constraints of limited equipment capacity and inferior part quality, a new scheme was proposed as shown in Fig. 5(c).

\section{Hydroforming process of blank bulging by turning the upside down}

As discussed below, the tonnage needed in case (b) exceeds the available capacity of the equipment, so this case could not be demonstrated. In order to compare the pros and cons of the cases (a) and (c), numerical simulations for the hydroforming process of corrugated sheet were carried out. In order to facilitate the comparison, the same meshes were used in the simulations for the two schemes. Elements with same positions were selected for the two blanks used in both of the schemes corresponding to zones I, II, III, IV, V on the component as illustrated in Fig. 4. For case (a), these selected elements are marked as A, B, C, D, E, and for case (c), these elements are marked as A1, B1, C1, D1, E1 for as displayed in Fig. 6. Charts for changing tendency of equivalent strains during the forming process for both of the schemes are shown in Fig. 7 and Fig. 8. 
ICNFT 2015

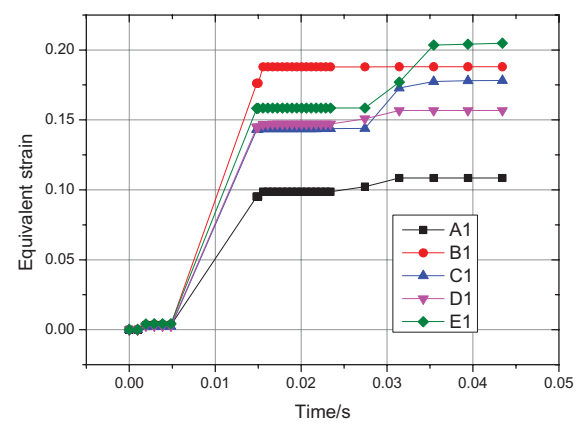

Figure 8. Effective strain variation curves of each region in the new hydroforming process.

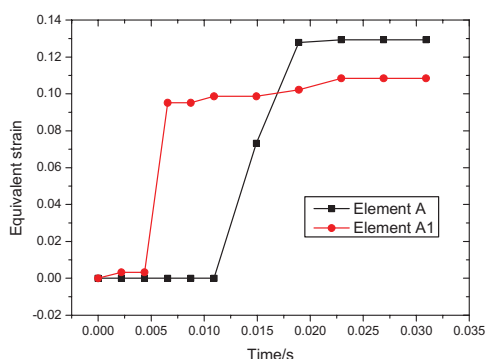

(a) Region I

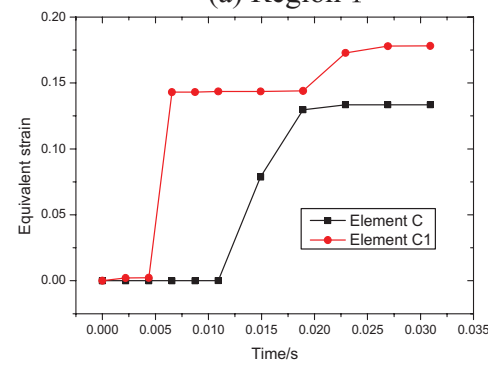

(c) Region III

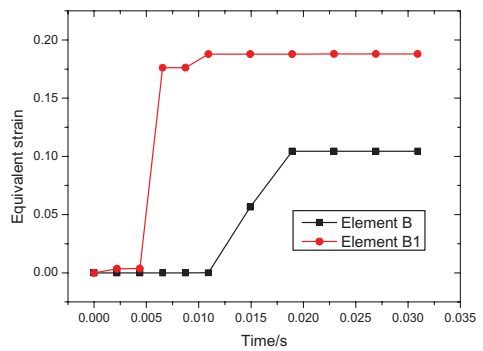

(b) Region II

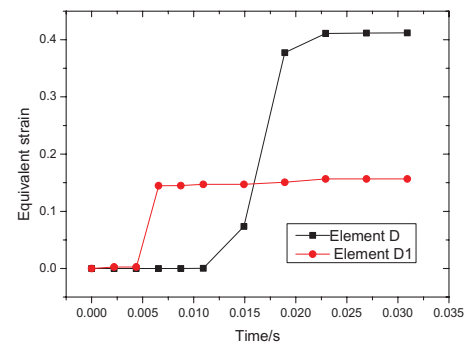

(d) Region IV

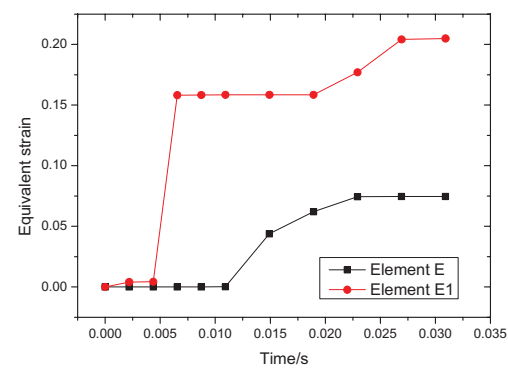

(e) Region V

Figure 9. Comparison of equivalent strain between two different forming process on each regions.

From Fig. 7, it can be observed that the equivalent strain of element D rises sharply in the interval $0.015 \mathrm{~s}$ to $0.02 \mathrm{~s}(2 \mathrm{MPa}-4 \mathrm{MPa})$, which is due to the action of pressure when the blank begins to bend along the underside round corner of punch. Under the combined effect of tensile and incidental tensile stresses caused by the wall bending, the thickness of the blank reduces rapidly which leads to its rupture 


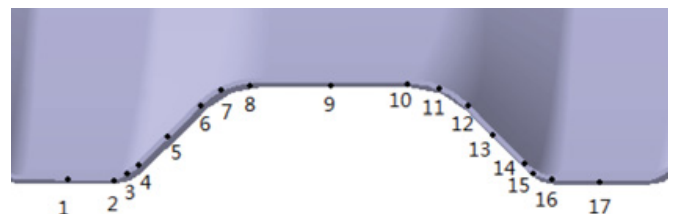

Figure 10. The measuring points.

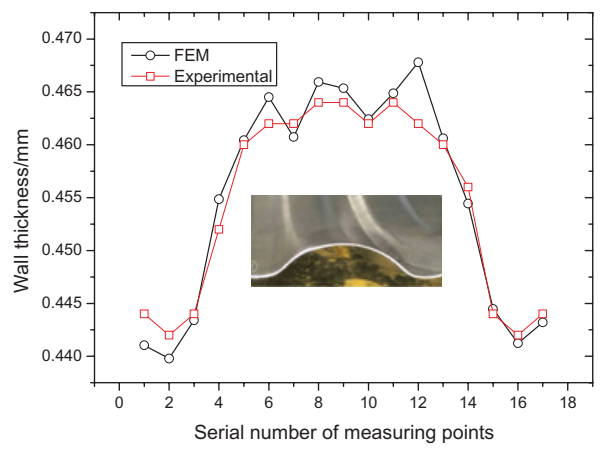

Figure 11. Wall thickness variations of experiments and FEM.

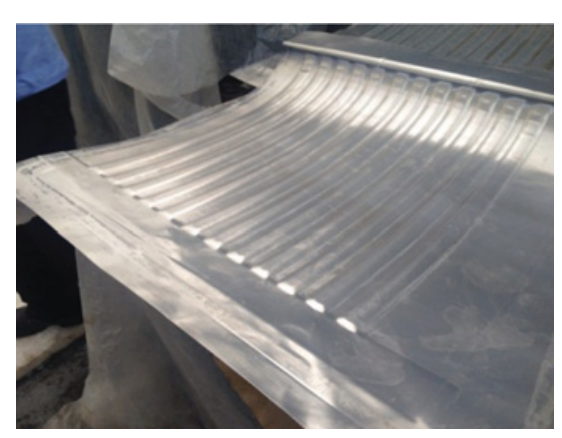

Figure 12. Part formed by scheme (c).

as shown in Fig. 9(a). While the deformation of the other elements is more uniform, the equivalent strain is about 0.15 . In Fig. 8, the interval from 0 to $0.025 \mathrm{~s}$ is the preforming stage, and the interval from $0.025 \mathrm{~s}$ to $0.045 \mathrm{~s}$ is the reforming stage of the blank for case (c). During the preforming stage, the deformation of element $\mathrm{B} 1$ is the highest at a pressure of $4 \mathrm{MPa}$ because this element lies in the bending zone. However, the biggest equivalent strain is only 0.18 due to the wider bending radius. At the reforming stage, due to the influence of the deformation already carried out during preforming phase and the size of the meshes, the elements $\mathrm{C} 1$ and $\mathrm{E} 1$ transform from bending to straight resulting in a considerable change in equivalent strains of these elements. From the Fig. 7 and Fig. 8 we can also find that the deformation of blank in case (c) is more uniform than case (a).

In order to compare the two schemes in more detailed, the equivalent strains of the same zones for these schemes have been compared separately, as shown in Fig. 9(a-e). From these figures, it can be seen that the deformation of elements B1, C1, D1in case (c) is considerably improved and the equivalent strains of these elements increase up to $79 \%, 33 \%$, and $124 \%$ as compared with the elements B, C, D in case (a). The deformation of element D1 which is the dangerous region in case (a) is reduced with $61 \%$ reduction in its equivalent strain. From the above results and discussion, it is evident that the deformation capacity of each region in case (c) is significantly improved effectively as compared with case (a).

From the FEM simulation of case (c), the optimal parameters considering minimum wall thickness distribution were obtained for experiments such that for the preforming process the clearance was $0.65 \mathrm{~mm}$ and internal pressure was $8 \mathrm{MPa}$ and for the reforming phase, the clearance was $0.65 \mathrm{~mm}$ and internal was $6 \mathrm{MPa}$. The component formed by case (c) is shown in Fig. 12. The component was sliced and the wall thickness was measured and compared with the FEM results as depicted in Fig. 11. It can be seen that the experimental results are in agreement with FEM study.

\section{Conclusions}

The active hydroforming process of corrugated sheet had been investigated using theoretical calculations, numerical simulations and process experiments. The following conclusions have been obtained from the present investigation: 


\section{ICNFT 2015}

1. For the shallow drawing, large deformation and complex components such as corrugated sheets are suitable for active hydroforming process.

2. The sheet bulging method of turning the blank upside down (case c) was proposed. This method is able to produce enhanced deformations with improved wall thickness/forming capacity and can prevent the failure due to rupture.

\section{References}

[1] Bark L.W., Yaniv G., Cost-effective production of helicopter energy-absorbing subfloor structures with advanced thermoplastic composites[C] the American Helicopter Society 49th Annual Forum (1993): 519-529

[2] L.H. Lang, Z.R.Wang et al., Hydroforming highlights: sheet hydroforming and tube hydroforming. Journal of materials processing technology 151 (2004) 165-177

[3] Lang L.H., Danckert J., Nielsen K.B., Study on Hydromechanical Deep Drawing with uniform Pressure onto the Blank[J]. International Journal of Machine Tools and Manufacture, (2004) 44 (5): 495-502

[4] H.J. Becker, G. Bensman, Further Development in Hydro mechanical Deep Drawing[C]. Developments in the Drawings of Metals on Metal Society of London (1983): 272-278

[5] Meng Bao, Min Wan et al., Influence of cavity pressure on hydrodynamic deep drawing of aluminum alloy rectangular box with wide flage. International journal of mechanical sciences $\mathbf{7 7}$ (2013) 217-226

[6] Ho Choi, Muammer Koç and Jun Ni, A Study on Warm hydro-mechanical deep drawing of lightweight materials. International journal of machine tools \& manufacture 47 (2007) 1752-1766 